\title{
Effects of rTMS Conditioning over the Fronto-parietal Network on Motor versus Visual Attention
}

\author{
Elisabeth Rounis $^{1}$, Kielan Yarrow ${ }^{1,2}$, and John C. Rothwel1 ${ }^{1}$
}

\begin{abstract}
Many studies have shown that visuospatial orienting attention depends on a network of frontal and parietal areas in the right hemisphere. Rushworth et al. [Rushworth, M. F., Krams, M., \& Passingham, R. E. The attentional role of the left parietal cortex: The distinct lateralization and localization of motor attention in the human brain. Journal of Cognitive Neuroscience, 13, 698-710, 2001] have recently provided evidence for a leftlateralized network of parietal areas involved in motor attention. Using two variants of a cued reaction time (RT) task, we set out to investigate whether high-frequency repetitive transcranial magnetic stimulation (rTMS; $5 \mathrm{~Hz}$ ) delivered "off-line" in a virtual lesion paradigm over the right or left dorsolateral prefrontal cortex (DLPFC) or the posterior parietal cortex (PPC) would affect performance in a motor versus a visual attention task. Although rTMS over the DLPFC on either side did not affect RT
\end{abstract}

performance on a spatial orienting task, it did lead to an increase in the RTs of invalidly cued trials in a motor attention task when delivered to the left DLPFC. The opposite effect was found when rTMS was delivered to the PPC: In this case, conditioning the right PPC led to increased RTs in invalidly cued trials located in the left hemispace, in the spatial orienting task. rTMS over the PPC on either side did not affect performance in the motor attention task. This double dissociation was evident in the first $10 \mathrm{~min}$ after rTMS conditioning. These results enhance our understanding of the networks associated with attention. They provide evidence of a role for the left DLPFC in the mechanisms of motor preparation, and confirm Mesulam's original proposal for a right PPC dominance in spatial attention [Mesulam, M. M. A cortical network for directed attention and unilateral neglect. Annals of Neurology, 10, 309-325, 1981].

\section{INTRODUCTION}

Many studies investigating attentional selection have identified specific cortical networks relating to spatial (Corbetta \& Shulman, 2002; Coull \& Nobre, 1998; Steinmetz \& Constantinidis, 1995) and feature-based attention (Liu, Stolnick, Serences, \& Yantis, 2003). Covert orienting of attention can be assessed in protocols in which spatial precues instruct subjects of the location of target stimuli on a screen; the precues allow subjects to covertly direct the focus of attention to the target's location before its appearance (Posner, Walker, Friedrich, \& Rafal, 1984). On a few trials in such protocols, the precue is invalid and the target appears on the opposite side of the screen. These trials are associated with slower response times, as visuospatial attention must be reallocated to the correct spatial location. Patients with lesions in the parietal cortex have a difficulty in "disengaging" covert visual attention from one focus to another (Posner et al., 1984). Neuroimaging studies have reported reliable activations in the fronto-parietal network relating to presentation of invalid trials (Nobre et al., 1997; Corbetta, Miezin, Shulman, \& Petersen, 1993).

\footnotetext{
${ }^{1}$ University College London, ${ }^{2}$ Institute of Cognitive Neuroscience, UCL, London
}

\section{The Dorsolateral Prefrontal Cortex and Motor versus Orienting Attention}

It has long been known that reaction times (RTs) can be shortened if subjects covertly attend to the motor response that they prepare to make (La Berge, LeGrand, \& Hobbie, 1969). In the 1980s, Rosenbaum used precues to indicate specific parameters of aimed hand movements such as their direction and extent. He showed that the use of precues could reduce RTs in a manner consistent with the partial programming of motor responses. Twenty years later, Rushworth, Ellison, and Walsh (2001), Rushworth, Krams, and Passingham (2001), and Rushworth, Nixon, Renowden, Wade, and Passingham (1997) were able to use tasks in which precues prepared subjects to move in a particular direction. They referred to the process the brain uses to perform these tasks as "motor attention" to distinguish it from visual attention. They have shown that precues can be used to covertly attend to or prepare for particular movements (Rushworth et al., 1997). As in visual attentional tasks, when the precue is valid, there is an RT shortening, but when a precue is invalid, responses are slower, presumably because a new response must be prepared (see also Stelmach \& Teulings, 1984). The parietal lobe has recently been implicated in the ability 
to perform invalidly precued trials in motor attention tasks (Rushworth, Ellison, et al., 2001).

Most studies performed so far have investigated the role of the parietal cortex in motor and visual attention (Rushworth, Krams, et al., 2001). However, fMRI evidence has suggested that the lateral prefrontal cortex also plays a crucial role in shifting attention, particularly relating to selecting task-relevant information (Brass \& von Cramon, 2004). The primary aim of this study was to investigate the roles of the right and left dorsolateral prefrontal cortices (DLPFCs) in the control of motor versus spatial attention. We chose to disrupt these areas on separate sessions with high-frequency $(5 \mathrm{~Hz})$ repetitive transcranial magnetic stimulation (rTMS) conditioning in an "off-line" virtual lesion paradigm (Jahanshahi \& Rothwell, 2000). Previous studies have shown that $5 \mathrm{~Hz}$ rTMS can induce efficient and long-lasting (up to $1 \mathrm{hr}$ ) increases in motor cortical excitability when applied to the motor (Peinemann et al., 2004) and dorsal premotor (Rizzo et al., 2004) cortices, after only 10 minutes of conditioning. In addition, preliminary data using a cued choice RT task, which included components of both motor and visual attention, suggested that $5 \mathrm{~Hz}$ rTMS over the right DLPFC compromised performance in invalidly cued trials. This conclusion was based on a comparison of RTs before versus after this conditioning paradigm (Siebner, Pesenti, Rizzo, \& Rothwell, 2003).

Two tasks were designed which separately tested performance when cues directed either motor attention or spatial attention prior to a go signal. Both tasks consisted of a random presentation of frequent validly and infrequent invalidly cued trials in which subjects were asked to respond with a finger press as soon as they saw a subsequent target (Figure 1). In the spatial attention task, subjects had to press their right index finger in response to a lateralized target. In the motor attention task, they had to press either their right or left index fingers depending on the direction instructed by a central target. The proportion of valid trials (80\%) exceeded that of the invalid trials (20\%) in order to ensure that subjects actually made use of the cues to direct attention or prepare a motor response. Changes in performance in these tasks was assessed in two groups of healthy volunteers who underwent right and left DLPFC conditioning in separate sessions to assess the contribution of these areas in motor and/or spatial reorienting attention.

\section{The Role of the Posterior Parietal Cortex in Directed Attention and Unilateral Neglect}

An additional set of experiments tested the effects of conditioning the right and left posterior parietal cortex (PPC) in a further two groups of subjects in the same tasks. Lesions within the right PPC are typically associated with deficits in directed attention and unilateral neglect (Mesulam, 1981). Neglect patients exhibit prob- lems in judging or responding to information on the contralesional side of space. Many studies involving rTMS have confirmed the crucial role of this area in spatial attention (Ellison, Schindler, Pattison, \& Milner, 2004; Bjoertomt Cowey, \& Walsh, 2002; Ashbridge, Walsh, \& Cowey, 1997). We wanted to confirm that the effects obtained by Rushworth, Ellison, et al. (2001) in their spatial attention task with parietal cortex "virtual lesions" could be replicated in our task.

\section{METHODS}

\section{Subjects}

Thirty-four right-handed healthy volunteers, with no history of neurological disorders or head injury, were recruited from the database of volunteers at the Functional Imaging Laboratory, Institute of Neurology, University College London, UK. They were divided into two groups of subjects allocated to one of two separate experiments: Experiment 1 (motor attention task) and Experiment 2 (spatial attention task). Subjects in Experiment 1 were further subdivided into two subgroups according to whether they were stimulated over the DLPFC (Group 1) or the PPC (Group 2)-Group 1 included 9 volunteers ( 1 woman, 8 men; mean age $=$ $35.6 \pm 6.7$ years); Group 2 included another 9 volunteers ( 4 women, 5 men; mean age $=34.8 \pm 7.1$ years). Subjects in Experiment 2 were also subdivided into two groups stimulated over either the DLPFC (Group 1: 8 subjects, 3 women, 5 men; mean age $=33.6 \pm 4.3$ ) or the PPC (Group 2: 8 subjects, 2 women, 6 men; mean age $=31.8 \pm 6.7$ ). Written informed consent was obtained from all participants. The study was approved by the joint ethics committee for the National Hospital for Neurology and Neurosurgery (UCLH NHS Trust) and the Institute of Neurology (UCL).

\section{r'TMS Conditioning}

Subjects from each group completed two sessions on separate days for each experiment, in which they received rTMS either on their right or left sides. The order in which they received these sessions was counterbalanced across subjects. Prior to rTMS, the "motor hot spot" was defined functionally as the point of maximum evoked motor response in the slightly contracted right first dorsal interosseous (FDI). Active motor threshold (AMT) was defined as the lowest stimulus intensity that elicited at least five FDI twitches in 10 consecutive stimuli given over the "motor hot spot." In each rTMS session, 1800 biphasic stimuli, at a stimulation intensity of $90 \%$ of AMT, were given over the left or right DLPFC area for subjects in Group 1 or the left or right PPC area for subjects in Group 2, using a MagStim-rapid stimulator connected to four booster modules (MagStim, Whitland, Wales, UK). The subjects received six 1-min 


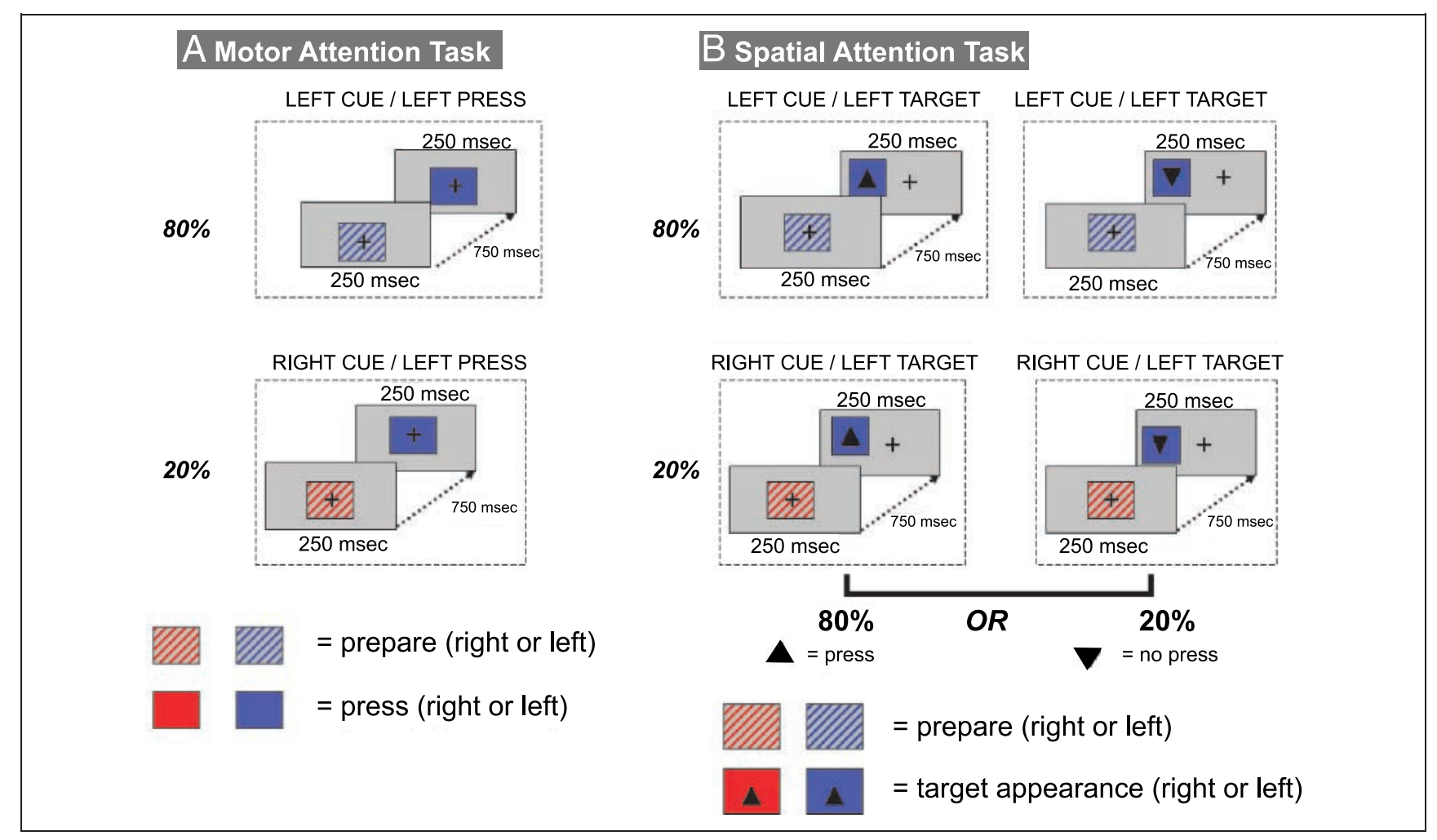

Figure 1. Precued choice reaction time task. This figure shows conditions pertaining to left-sided responses for the motor task or left-sided target appearance for the spatial attention task. Blue $=$ left side, Red $=$ right side. Note that the color rules were counterbalanced across subjects. (A) Motor attention task: Subjects maintained fixation of a central black cross against a gray background. A central warning precue was in the form of a colored square with a hashed fill. The color of the precue indicated whether subjects should prepare a response with their left or their right index finger. The target stimulus was another colored square of identical size to the precue but this time with a uniform (solid) fill. Subjects were asked to respond with the finger press corresponding to the color of the target as rapidly as possible and without making mistakes. In this task, all cues and targets were presented centrally and responses were made on two separate one-key button boxes (one for each hand) according to the color of the target. The number of trials in each block was 80 , with 20 two-second pauses inserted at random places in this sequence. Eighty percent of trials were validly cued and $20 \%$ were invalidly cued so that subjects who had prepared a motor response on one side now needed to press the opposite finger. Subjects performed three blocks: before, immediately after, and $10 \mathrm{~min}$ after rTMS conditioning. (B) Spatial attention task: The spatial attention task retained the stimulus timings and the color-direction mappings of the motor attention task, but differed from it in the following ways. The color of the precue (red or blue) indicated the location to which subjects should direct their spatial attention. The target stimulus consisted of a black outline triangle presented within a solid-colored square centered $10^{\circ}$ to either the left or the right of the fixation cross. The color of the target square was consistent with its side of presentation. Subjects were asked to respond with a right index finger press on a single one-key button box whenever they detected an upward-facing triangle, but not to respond to the occasional downward-facing triangles. Block length was 100 trials presented in a pseudorandom order. Eighty percent of trials were validly cued and $20 \%$ were invalidly cued so that subjects who were expecting a visual target on one side were presented with a visual target on the opposite side. Subjects performed three blocks: before, immediately after, and 10 min after rTMS conditioning.

trains of $5 \mathrm{~Hz}$ rTMS separated by an intertrain interval of $1 \mathrm{~min}$. A standard figure-of-eight-shaped coil (Double 70-mm Coil Type P/N 9925, MagStim) was used to deliver rTMS. The coil was positioned with the handle at $45^{\circ}$ to the sagittal plane. The current flow of the initial rising phase of the biphasic pulse in the TMS coil induced a current flowing from posterior-to-anterior in the underlying cortex.

The site of rTMS stimulation for subjects in Group 1 (DLPFC) was located $5 \mathrm{~cm}$ anterior to the "motor hot spot" on a line parallel to the mid-sagittal line. This is the same location of DLPFC stimulation used in a recent study by Mottaghy, Gangitano, Sparing, Krause, and Pascual-Leone (2002). Structural T1-weighted scans with fiducial markers located over the point of DLPFC stimulation bilaterally have confirmed that this is an accurate location for that area (Rounis et al., in press). The site of rTMS stimulation for subjects in Group 2 (PPC) was located over the left and right posterior parietal areas, respectively, identified by the $\mathrm{P} 3$ and $\mathrm{P} 4$ electrode positions of the 10-20 EEG system. Previous studies have used these sites to stimulate the PPC (Ellison et al., 2004; Bjoertomt et al., 2002; Oliveri et al., 2000; Ashbridge et al., 1997) with MRI evidence (Fierro, Brighina, Oliveri, \& Caltagirone, 2000), suggesting that these sites of stimulation are located near sites shown to be activated in tasks involving spatial attention such as in conjunction search (Corbetta et al., 1991). 
The contracted FDI was used to define the AMT because TMS-evoked twitches are clearly visible and the FDI has a threshold below other intrinsic hand muscles. This ensured that the intensity used for rTMS was below motor threshold for all hand muscles. The use of subthreshold intensity reduced the spread of stimulation away from the targeted site (Munchau, Bloem, Irlbacher, Trimble, \& Rothwell, 2002; Gershlager et al., 2001; Pascual-Leone, Valls-Sole, Wassermann, \& Hallett, 1994).

In this study, any changes in performance found following $5 \mathrm{~Hz}$ rTMS conditioning in the stimulated sites should reflect alterations in the separate motor and attentional networks involved in the different tasks. This might either provide evidence of a direct role of the stimulation sites in these networks or of changes in effective connectivity of these sites of stimulation with areas functionally connected to them during performance of these tasks (Rounis et al., 2005). The exact nature of these connectivity changes is beyond the scope of this study and will be addressed in future functional neuroimaging experiments.

Repetitive transcranial magnetic stimulation conditioning was performed "off-line" (Mottaghy et al., 2002; Jahanshahi \& Rothwell, 2000) between the behavioral testing sessions. The total duration of stimulation was $11 \mathrm{~min}$ and behavioral testing was initiated immediately after the end of the stimulation period. Previous studies have shown that this rTMS protocol induces physiological effects that can last for up to $1 \mathrm{hr}$ after the end of the stimulation (Rounis et al., 2005; Peinemann et al., 2004).

\section{Experiment 1: Motor Attention Task}

Figure 1A describes the motor attention task used in this study. Stimuli were presented against the gray background of a CRT monitor refreshing at $120 \mathrm{~Hz}$ (screen $40 \mathrm{~cm}$ away from subject's eyes). Responses were made on two separate one-key button boxes (one for each hand). Subjects initially fixated a central black cross subtending $1.5^{\circ}$ of visual angle. Each trial began with the 250 -msec presentation of a warning precue in the form of a colored square with a hashed (as opposed to uniform) fill. The precue appeared at the center of the screen, subtending $7.5^{\circ}$ visual angle, with the fixation cross remaining visible at its central point. The color of the precue indicated whether subjects should prepare a response with their left or their right index finger. Subjects were randomly allocated to one of two subgroups, for which blue represented left-hand movement and red a right-hand movement or vice versa. After the warning precue disappeared, the fixation cross was presented alone for $750 \mathrm{msec}$, followed by the presentation of a target stimulus (on screen for $250 \mathrm{msec}$ ). The target stimulus was another colored square of identical size to the precue but this time with a uniform (solid) fill. Subjects were required to make a speeded response to this stimulus. In $80 \%$ of the trials, the color of the target was the same as that of the precue (valid precue): that is, a blue-hashed square was followed by a blue square or a red-hashed square was followed by a red square. In 20\% of the trials, however, the color of the precue was different from that of the target (invalid trials). In this case, the subjects had to move the opposite index finger to the one they had prepared to move given the warning precue. Subjects were asked to respond with the finger press corresponding to the color of the target as rapidly as possible and without making mistakes. Following the disappearance of the target, the fixation cross was presented alone for $750 \mathrm{msec}$ before the next trial began; hence, each trial was $2 \mathrm{sec}$ in duration. If subjects failed to respond in the 1-sec period between the onset of the target and the beginning of the subsequent trial, they were considered to have made an error of omission. Trials were presented in a pseudorandom order, with 80 trials per block. In addition, twenty 2 -sec-long pauses were inserted at random places in this sequence, giving rise to unpredictable pauses between trials. Subjects performed an initial practice block of 80 trials prior to each session. They then performed three blocks of 80 trials before rTMS conditioning, three blocks of 80 trials immediately after the conditioning, and another three blocks of 80 trials 10 min after the conditioning.

\section{Experiment 2: Spatial Attention Task}

The spatial attention task (Figure 1B) retained the stimulus timings and the color-direction mappings of the motor attention task, but differed from it in the following respects. The central fixation cross now subtended only $1^{\circ}$ of visual angle. The centrally presented square colored (hashed) precue was also reduced in size, subtending $4^{\circ}$ of visual angle. The color of the precue now indicated the location to which subjects should direct their spatial attention. For this task, the subsequent target stimulus consisted of a black outline triangle (subtending $2^{\circ}$ ) presented within a solid-colored square (subtending $4^{\circ}$ ) centered $10^{\circ}$ to either the left or right of the fixation cross. The color of the target square was consistent with its side of presentation, reflecting the color-direction mapping assigned to the current subject. Subjects were required to respond with a right index finger press on a single one-key button box whenever they detected an upward-facing triangle.

The precue indicated the correct direction of the target's appearance in $80 \%$ of the trials (valid trials). In $20 \%$ of the trials, the precue indicated the opposite direction (left when the target appeared right or vice versa). In addition, a small proportion of all valid and invalid trials (20\%) were catch trials, where the target presented consisted of a downward-pointing triangle. In these trials, subjects were required to withhold their response. This type of catch trial has been used in 
previous studies (Bartolomeo, D'Erme, Perri, \& Gainotti, 1998), particularly when subjects have been required to simply press a single key as fast as possible in response to targets appearing in their peripheral visual field without having to reach or change hands for their response (Husain, Mattingley, Rorden, Kennard, \& Driver, 2000; Mattingley, Husain, Rorden, Kennard, \& Driver, 1998). They were devised to avoid the possibility of subjects responding to a fixed time interval (Bartolomeo et al., 1998). The requirement to discriminate (rather than merely to detect) a peripheral target also makes the spatial attention task slightly more demanding than a typical Posner task. We hoped that this would increase the size of the cueing effects in the spatial attention task, making them comparable with the large cueing effects we anticipated in the motor attention task.

Block length was increased to 100 trials to accommodate the additional catch trials. Subjects were required to respond as quickly and accurately as possible every time they saw the upward-pointing triangle and were asked to keep fixating on the central fixation cross. Their eye movements were monitored with an infrared eyetracker (Applied Science Laboratories Eye-trac model 310) and sampled at $200 \mathrm{~Hz}$ (12-bit A/D card; National Instruments DAQ 1200). If the eyes strayed horizontally from the fixation point by more than $3^{\circ}$ of visual angle, the trial was repeated. Subjects performed an initial practice block of 100 trials prior to each session. They then performed three blocks of 100 trials before rTMS conditioning, three blocks of 100 trials immediately after the conditioning, and another three blocks of 100 trials 10 min after the conditioning.

\section{Statistical Analysis}

As mentioned above, all participants were subdivided according to the experimental task (motor or spatial orienting attention) and the site of stimulation (DLPFC or PPC). Those who failed to show any validity effect (i.e., reacted no faster in validly cued than in invalidly cued trials) were excluded from the study (2 subjects on the spatial attention task, leaving a total of 14 subjects in two groups of 7 analyzed for this experimental task).

Separate analyses were performed in the DLPFC or PPC stimulated groups for each task (motor vs. orienting spatial attention). For each subject, the mean RT, standard deviation, and error rates were calculated in each condition. Subjects made two kinds of errors in each task. These were: errors of omission and direction in the motor attention task; errors of omission and commission (responding to downward triangles) in the spatial attention task. However, the errors of omission were extremely infrequent $(<1.1 \%$ of total trials). They were therefore combined with the errors of direction in the motor attention task and the errors of commission in the spatial attention task to give a single error score in each task. RTs larger than 3 standard deviations from the mean were excluded on a trial-by-trial basis. Error trials were also excluded from the RT analysis.

Data were entered into four-way repeated-measures analyses of variance (ANOVAs), using the GreenhouseGeisser correction where appropriate. The factor side of rTMS conditioning had two levels (right vs. left), the factor side of motor response (for the motor attention task) or side of stimulus appearance (for the spatial attention task) had two levels (right vs. left), the factor cue validity had two levels (valid vs. invalid) and the factor time had three levels [pre (i.e., prior to rTMS conditioning), post1 (0-10 min after rTMS conditioning), and post2 (10-20 min after rTMS conditioning)]. When an interaction $F$ value was significant, follow-up ANOVAs and/or post hoc paired-sample $t$ tests were performed. Data were analyzed using SPSS 11.5 (SPSS, Chicago, IL, USA). Results were considered significant if the $p$ value was $<.05$.

\section{RESULTS}

No subject reported adverse side effects during the course of the study. Mean AMT for rTMS over the right FDI was $46 \%$, ranging from $36 \%$ to $59 \%$ of maximum stimulator output, and over the left FDI was 49\%, ranging from $37 \%$ to $61 \%$ of maximum stimulator output. The maximum stimulator output with the coil and stimulator used was approximately $1.8 \mathrm{~T}$.

\section{Experiment 1: Motor Attention Task}

Effects of $5 \mathrm{~Hz}$ rTMS over the DLPFC

The effect of $5 \mathrm{~Hz}$ rTMS over the right or left DLPFC on response times (Figure 2A) was examined with a fourway repeated-measures ANOVA. A significant main effect of cue validity revealed that RTs on invalid trials were slower than on valid trials [main effect of cue validity, $F(1,8)=32.557, p<.001]$. The main effect of time showed a learning trend with decreasing RTs in the post 1 and post 2 blocks of trials $[F(2,16)=3.04, p=$ .063]. The only significant interaction between factors induced by $5 \mathrm{~Hz}$ rTMS was revealed by a three-way interaction [Side of rTMS stimulation $\times$ Cue validity $\times$ Time interaction: $F(2,16)=6.778, p=.023$ ]. RTs in both hands were slower in invalidly cued trials but not validly cued trials after $5 \mathrm{~Hz}$ rTMS to the left DLPFC (see Figure 2A: average RT in invalidly cued trials, right and left hand responses combined, was increased by 21.5 msec after left DLPFC conditioning). This was confirmed by post hoc $t$ tests showing that RTs in invalidly cued trials were slowed in the first $10 \mathrm{~min}$ following $5 \mathrm{~Hz}$ rTMS to the left DLPFC $[t(8)=-2.414$, $p=.027]$ compared to RTs in invalidly cued trials measured prior to the conditioning (Figure 2A). Conversely, RTs in invalidly cued trials decreased with time following $5 \mathrm{~Hz}$ rTMS to the right DLPFC $[t(8)=2.588, p=$ 


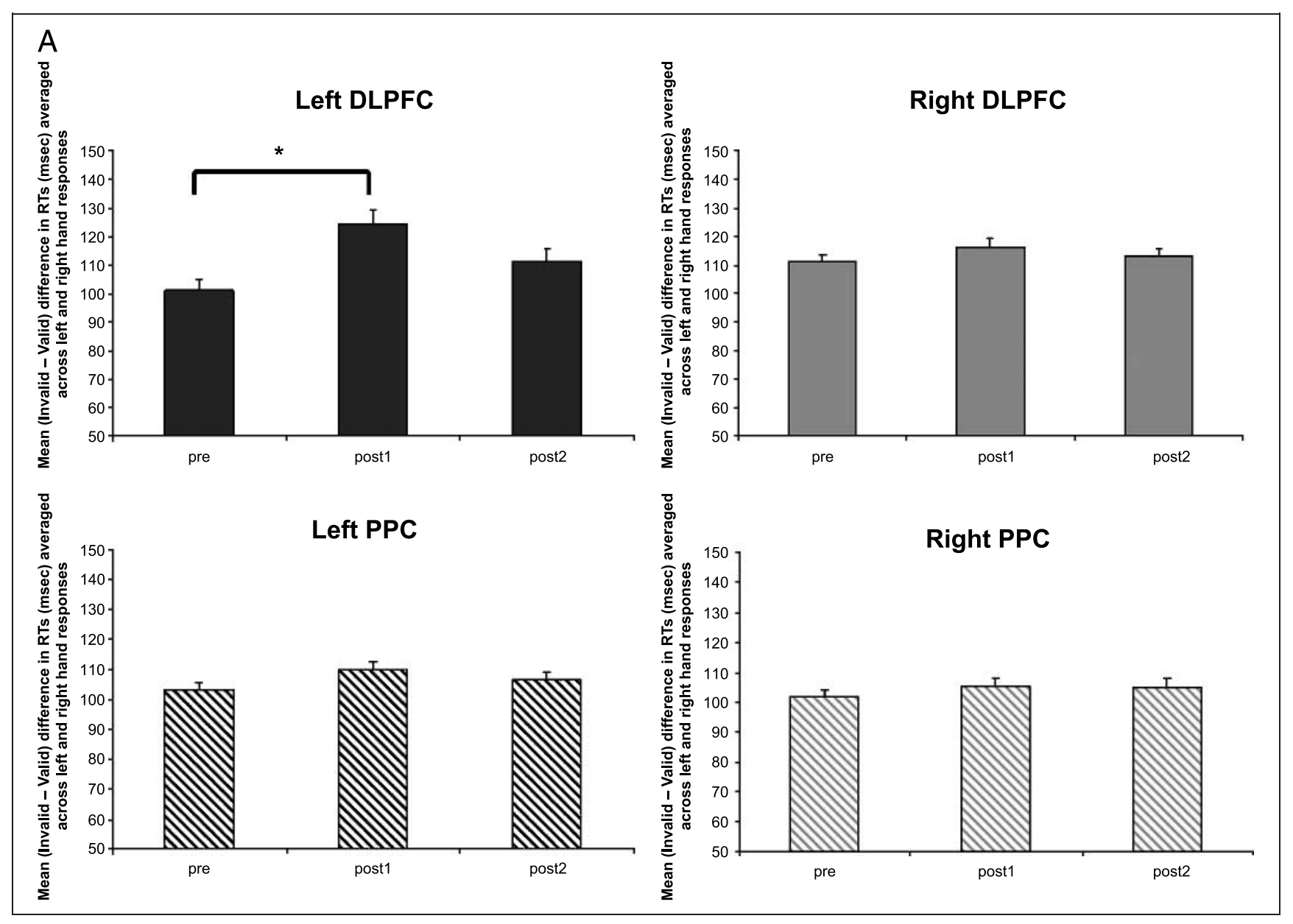

Figure 2. Effects of $5 \mathrm{~Hz}$ rTMS on the (A) motor and (B) spatial attention tasks (with error bars showing standard deviations).

$\mathrm{DLPFC}=$ dorsolateral prefrontal cortex; PPC $=$ posterior parietal cortex. Data from the group of subjects with DLPFC conditioning are presented on the upper panels. Data from the PPC conditioning group are presented on the lower panels. The side of conditioning (left vs. right) is located on the corresponding left or right side in each panel. Pre: Baseline measurement before rTMS conditioning; Post1: 0-10 min after rTMS conditioning; Post2: 10-20 min after rTMS conditioning.

(A) Motor attention task: In both groups of subjects, RTs in validly cued trials were significantly lower than RTs in invalidly cued trials (DLPFC group: valid RTs $=289$, invalid RTs $=403.4$; PPC group: valid RTs $=277.7$, invalid RTs $=411.65)$. Histograms show the mean differences in RTs

between valid and invalid trials (invalid - valid) averaged across left- and right-hand responses at each measurement time (pre, post1, and post2). Results are shown in separate panels for each session representing site and side of stimulation.

Upper panel: Left DLPFC conditioning led to slower RTs in invalidly cued trials in the first 10 min after stimulation compared to the prestimulation baseline, regardless of whether the left (12 msec increase in invalidly cued RTs) or right ( 31 msec increase in invalidly cued RTs) hand was used. The average increase in invalid-valid RT difference was $24.36 \mathrm{msec}$ 0-10 min after stimulation compared to baseline. This contrasts with the faster RTs found in all other conditions at this time point (i.e., following the right DLPFC, left and right PPC stimulation). RTs came back to baseline levels of performance as in the "pre" test block in the next 10-20 min after left $5 \mathrm{~Hz}$ DLPFC conditioning.

Lower panel: Stimulation over the left or right PPC did not differentially affect RTs in the motor attention task.

.019]. It seems that left-sided rTMS affected left- and right-handed responses equally, as no significant main effects or interactions were revealed for the factor "side of motor response."

As mentioned in the Methods section, we also looked for differences in the standard deviations (SDs) of response times (see Figure 3). Given that the factor "side of motor response" showed no significant main effect or interactions, responses pertaining to the left or right hand were collapsed together. The results were entered into in a three-way ANOVA, with the factors side of rTMS conditioning, cue validity, and time. A significant main effect of cue validity was found $[F(1,8)=5.03, p=$ $.047]$, and a significant two-way interaction of Side of rTMS conditioning $\times$ Time $[F(2,16)=6.706, p=.024]$. However, despite an apparently opposite effect of left DLPFC stimulation on the $S D$ of invalidly cued trials compared to validly cued trials, the three-way interaction (Side of rTMS conditioning $\times$ Cue validity $\times$ Time) was not significant. Figure 3 shows that the $S D$ of invalidly cued trials was increased in the first $10 \mathrm{~min}$ following $5 \mathrm{~Hz}$ rTMS compared to preconditioning only when the left DLPFC was targeted, whereas the opposite was true for validly cued trials and for trials that 


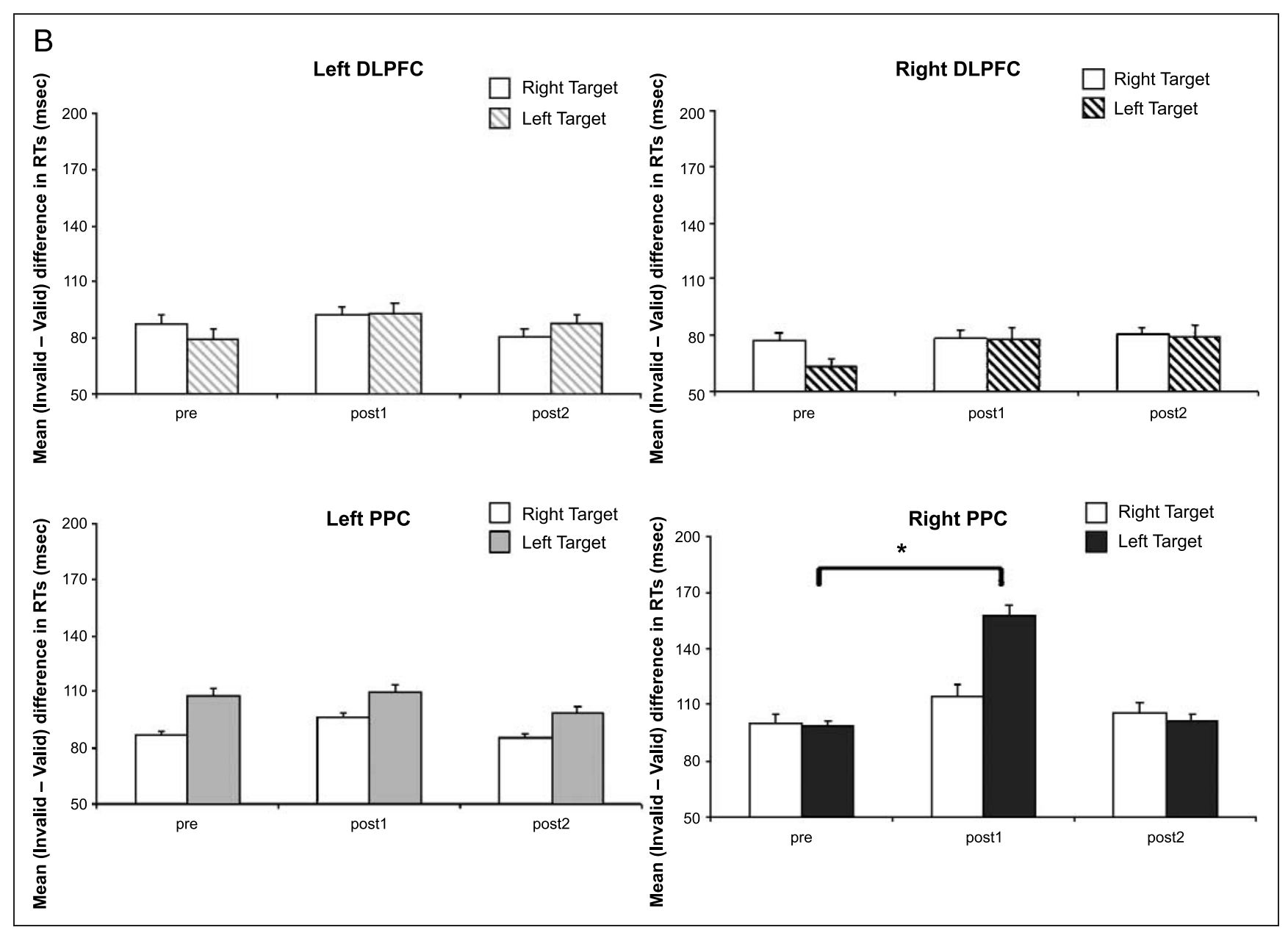

Figure 2. (continued). (B) Spatial attention task: Both groups of subjects showed a difference in RTs between validly and invalidly cued trials (invalid being slower than valid: DLPFC group: valid RTs $=319.75$, invalid RTs $=400.91 ;$ PPC group: valid $\mathrm{RTs}=299.1$, invalid RTs $=404$ ). Histograms show the mean differences in RTs between valid and invalid trials (invalid - valid) separately in the left and right target appearance conditions at each measurement time (pre, post1, and post2). Left Target = (right cue, left target appearance) - (left cue, left target appearance) Right Target $=$ (left cue, right target appearance) - (right cue, right target appearance)

Upper panel: $5 \mathrm{~Hz}$ rTMS to the right or left DLPFC had no differential effect upon performance in either right or left invalid-valid conditions. Lower panel: A significant effect was revealed in the "Left Target" condition at post1 (0-10 min) following right PPC conditioning (lower right panel). Stimulation of the right PPC led to a selective increase in RTs in invalidly cued trials in which subjects were cued to the right but the target subsequently appeared in the left hemifield. This increase $(42.8 \mathrm{msec})$ only lasted for the first $10 \mathrm{~min}$ after stimulation, returning to baseline levels in the subsequent block of $10 \mathrm{~min}$ (post2).

followed right DLPFC conditioning. Because the mean RT data did reveal a significant three-way interaction (see above), we felt justified to perform follow-up $t$ tests to confirm the effect of left DLPFC stimulation on the $S D$ of invalidly cued trials [post hoc $t$ test for invalid trials: post1 vs. pre: $t(8)=-2.992, p=.017$, post 2 vs. pre: $t(8)=-0.567, p=.588$; post hoc $t$ test for valid trials: post1 vs. pre: $t(8)=1.135, p=.225$, post 2 vs. pre: $t(8)=1.521, p=.125]$. In short, rTMS to the left DLPFC induced an increase in invalidly cued trials' RTs and the standard deviation of these RTs in the first 10 min following the end of stimulation in this motor attention task.

Although $5 \mathrm{~Hz}$ rTMS to either the left or right DLPFC did not lead to any significant changes in error rates before versus after conditioning, the four-way repeated- measures ANOVA did reveal a main effect of cue validity $[F(1,8)=16.164, p=.004]$ as the proportion of errors in invalidly cued trials was higher (8.8\%) than in validly cued trials $(2.9 \%)$, which rules out a speed/accuracy tradeoff for this specific effect.

\section{Effects of $5 \mathrm{~Hz}$ rTMS over the PPC}

Differences in RTs between invalid and valid trials pooled across right- and left-hand responses are shown in Figure 2A (lower panel). The four-way repeatedmeasures ANOVA carried out on RTs for this task and site of stimulation revealed simple main effects of cue validity $[F(1,8)=35.525, p=.001]$ and time $[F(1,8)=$ $6.353, p=.033]$. As in the previous group, invalid trials were slower than valid trials. The lack of any effect 


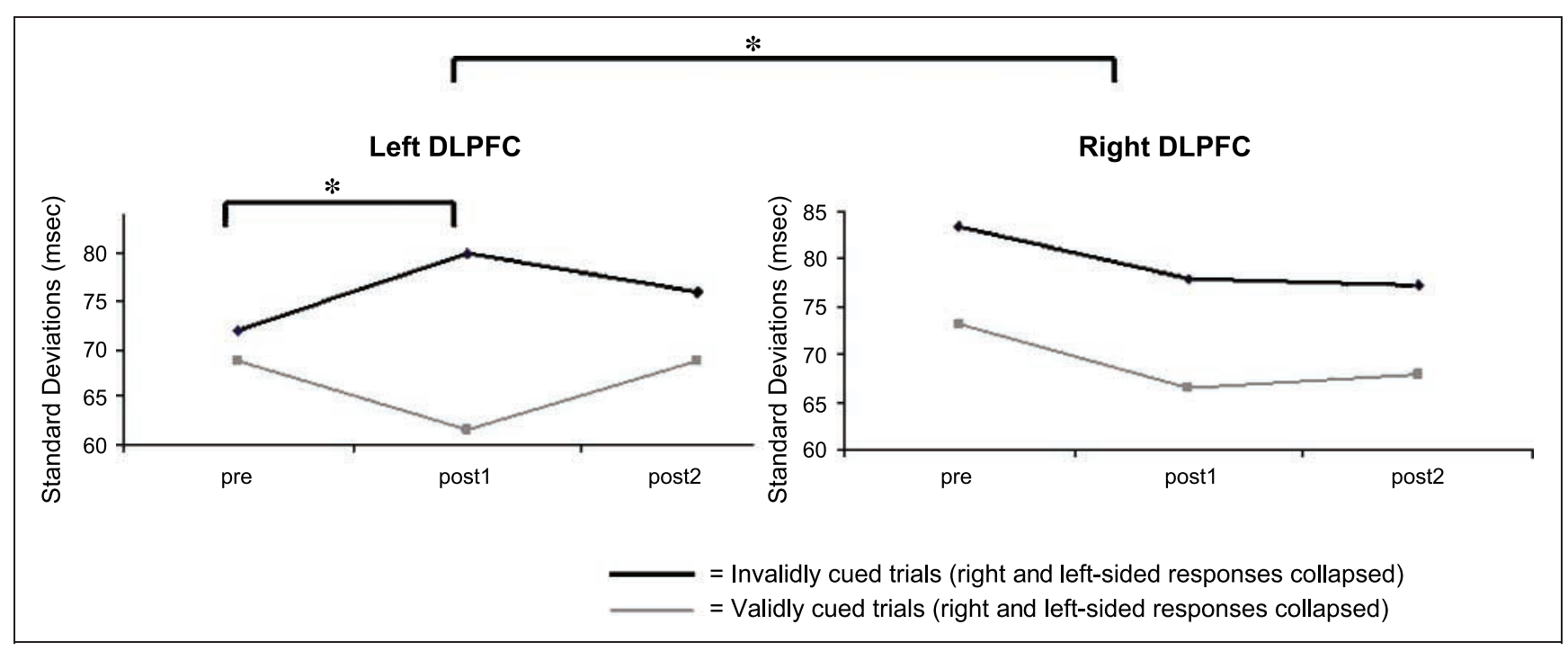

Figure 3. Effects of $5 \mathrm{~Hz}$ rTMS over the DLPFC on SDs of RTs in the motor attention task: SDs in the invalidly cued trials immediately after left-sided rTMS conditioning were increased compared to validly cued trials or right-sided rTMS conditioning of the DLPFC. Pre: Baseline measurement before rTMS conditioning; Post1: 0-10 min after rTMS conditioning; Post2: 10-20 min after rTMS conditioning.

following PPC conditioning either to the right or to the left sides, as indexed by absent task-related interactions in the motor attention task, was expected and confirms the results of Rushworth, Krams, et al. (2001). They showed that the center for motor attention in the parietal areas is left lateralized and located more anterior to the PPC, within the supramarginal gyrus (SMG).

No significant interactions were found in RTs or standard deviations. As in the previous group, error rates showed a main effect of cueing consistent with the RT data $[F(1,8)=32.068, p<.001]$. Error rates were $8.5 \%$ in invalid trials and $3.05 \%$ in valid trials. In addition, an interaction between side of motor response and cue validity $[F(1,8)=5.623, p=.045]$ indicated that subjects in this group seemed to make more errors when cued to the right and having to make a left-hand response than vice versa. Given that the numbers of errors were so small and that this effect was not found in the previous (DLPFC) group, it is difficult to draw any definite conclusions about this effect, which, in any case, was not affected by the rTMS conditioning.

\section{Experiment 2: Spatial Attention Task}

Effects of $5 \mathrm{~Hz}$ rTMS over the DLPFC

A 5-Hz rTMS over either the left or the right DLPFC did not lead to any significant task-related interactions, thus indicating that conditioning over this site produced no significant site-specific changes in RTs (Figure 2B, upper panel). A four-way ANOVA revealed a simple main effect of validity, suggesting, as expected, that RTs in invalidly cued trials were longer than in validly cued trials $[F(1,6)=$ $81.872, p<.001]$ as well as a simple main effect of time (pre vs. post1 vs. post2), suggesting some session effect as the RTs decreased over the blocks of trials $[F(2,14)=$ 7.527, $p=.031]$. No other significant main effects or interactions were found in RTs or standard deviations.

A four-way repeated-measures ANOVA on the error rates in this task revealed a significant four-way interaction $[F(1,6)=8.593, p=.017]$ but no other effects. However, additional analysis with 2 three-way repeatedmeasures ANOVAs (one for each side of stimulation, left or right) showed no significant effects. In the absence of any significant effects in the post hoc ANOVAs, the above-mentioned interaction is difficult to interpret. It may simply have occurred by chance. Errors in this task comprised $4.3 \%$ of the total trials and seemed to be unaffected by cue validity (4.8\% valid trials, 3.8\% invalid, $n s$ ) or rTMS conditioning.

\section{Effects of $5 \mathrm{~Hz}$ rTMS over the PPC}

Figure 2B (lower panel) shows the effects of rTMS over the PPC on the spatial attention task. A four-way ANOVA revealed a number of significant effects including a significant four-way interaction $[F(1,5)=5.872, p=.05]$. We therefore ran 2 three-way ANOVAs testing each side of $5 \mathrm{~Hz}$ rTMS conditioning separately. RTs following left PPC conditioning did not show any significant interactions, the only significant effects being the main effects of validity $[F(1,6)=35.836, p=.001]$ and side of stimulus appearance $[F(1,6)=7.394, p=.035]$. However, the ANOVA for RTs with right PPC conditioning demonstrated a significant three-way interaction [Cue validity $\times$ Side of stimulus appearance $\times$ Time: $F(1,5)=8.291, p=.026]$ alongside a number of other significant effects. Further analysis of the right-sided stimulation data with 2 twoway ANOVAs testing valid and invalidly cued trials following 5 Hz rTMS PPC conditioning separately revealed 
a significant Side of stimulus appearance $\times$ Time interaction for invalidly cued trials only $[F(1,5)=12.206, p=$ $.012]$, whereas post hoc $t$ tests confirmed that conditioning the right PPC alters performance of invalidly cued trials in which subjects were cued to the right but where the target appeared to the left $[42.8 \mathrm{msec}$ increase in invalidly cued RT when target was presented in the left side of the fixation cross; post hoc $t$ test for left invalid trials: post1 vs. pre: $t(6)=-7.434, p<.001$ ], but not in invalidly cued trials in which subjects were cued to the left but the target appeared to the right [post hoc $t$ test for right invalid trials: post1 vs. pre $t(6)=-0.494$, $p=.438]$.

Analysis of $S D$ s and error rates did not reveal any significant interactions following $5 \mathrm{~Hz}$ rTMS conditioning to the PPC on either side. Error rates in this task were low (4.96\% for valid trials, $3.98 \%$ for invalid trials, no significant difference between the two).

\section{DISCUSSION}

We studied the effects of conditioning the right or the left fronto-parietal areas with $5 \mathrm{~Hz}$ rTMS on a motor versus a spatial attention task. Left DLPFC conditioning selectively increased RTs in invalidly cued trials in the motor attention task. Conditioning the right PPC selectively slowed RTs in the spatial attention task for invalidly cued trials when the target was presented to the left.

These results confirm that the type of "off-line" rTMS conditioning used in this study was a useful "virtual lesion" technique. In addition, they extend previous work on attentional selection in the context of motor or visual attention by providing evidence for a double dissociation between task modality and site of virtual rTMS lesion. Double dissociations of this kind provide strong evidence for the causal involvement of a neural region in a specific task by eliminating alternative explanations of single dissociations, such as differences in task sensitivity (Shallice, 1988). The organization of attention systems within the frontal and parietal cortex will be discussed separately in the following sections, illustrating the relevance of the behavioral effects obtained in this study.

\section{Visual Attention and the Selection of Task-relevant Information}

Evidence shows that different cortical networks might be involved in reorienting attention (Corbetta \& Shulman, 2002; Pollmann, 2001; Corbetta et al., 2000) compared to the context of selecting task-relevant information (Brass \& von Cramon, 2004; Liu et al., 2003; Banich et al., 2000; MacDonald \& Joordens, 2000). Rushworth, Krams, et al. (2001) and Rushworth et al. (1997) have argued this dissociation within the parietal cortex (Snyder, Batista, \& Andersen, 1997) by distinguishing between visual and response switching. Parietal lesion patients were tested in a task in which precues allowed subjects to covertly prepare subsequent cued hand movements as opposed to orienting eye movements. Patients with left parietal lesions, as opposed to patients with right parietal lesions or control subjects, were impaired in their ability to disengage the focus of motor attention from one hand movement to another (Rushworth et al., 1997). A functional neuroimaging study showed the exact locus of motor attention to be in the left SMG, anterior to the angular gyrus (ANG) in the parietal cortex (Rushworth, Krams, et al., 2001). The role of the left SMG in motor attention in healthy subjects was subsequently confirmed following disruption of the left anterior parietal cortex with on-line rTMS, which compromised performance in a motor attention task. Conversely, disruption of the right ANG impaired spatial reorienting to invalidly cued trials (Rushworth, Ellison, et al., 2001).

\section{Role of the Prefrontal Cortex in Motor and Spatial Attention: A Left Hemispheric Network for Response Selection}

Our findings of compromised performance in invalidly cued trials caused by left DLPFC rTMS conditioning only in the motor attention task extend results described by Rushworth, Ellison, et al. (2001), Rushworth, Krams, et al. (2001), and Rushworth et al. (1997) by suggesting a role for the left lateral prefrontal cortex in motor attention. We propose that this area forms a dynamic network with left anterior parietal regions, such as the left SMG (Rushworth, Ellison, et al., 2001; Rushworth, Krams, et al., 2001), in the control of response switching.

The process of response switching would seem to require subjects to both suppress a prepared response, and select and activate a new response. Assuming these subprocesses are serial, our RT deficit might have arisen from a problem with either or both of these. In a recent study by Mayr, Diedrichsen, Ivry, and Keele (2006), who examined the role of the right and left prefrontal cortex on task-set selection and inhibition, the authors found that patients with left frontal lesions show a global deficit in activating/retrieving a currently relevant task set, whereas right prefrontal cortex involvement was more related to task-set inhibition (see also Aron et al., 2004). In our data, we found a deficit following left DLPFC conditioning but not following right DLPFC conditioning. Although our motor task involved response switches rather than shifts of task set, Mayr's behavioral observations suggest that the RT deficit we observed might have resulted from a failure to rapidly activate or retrieve the new motor response for the uncued hand, rather than a failure to rapidly inhibit or deselect the cued motor response.

A preliminary behavioral study, using a task in which spatial and motor attention were not separated from each other (subjects had to respond with either a right or left index finger press to precued targets appearing on the corresponding right or left side of a fixation 
cross), suggested that right DLPFC conditioning leads to selective prolongation of RTs in invalid trials (Siebner et al., 2003). However, it was not clear whether rTMS had interfered with the process of shifting spatial attention or the process of shifting motor attention (i.e., preparing a new response) on invalid trials.

The present study extends those results by showing an effect when the left-lateralized fronto-parietal network, known to be dominant for motor preparation, was targeted in a task exclusively testing the ability of subjects to shift the direction of a prepared motor response. Our results suggest that the ability to shift spatial attention is not impaired following $5 \mathrm{~Hz}$ rTMS to the right DLPFC, so the deficit found in Siebner et al.'s study is instead likely to have reflected the task's motor demands. Subtle differences existed in the nature of the attentional precues used in the two studies: They were endogenous in the current research and exogenous in Siebner et al.'s study. The nature of the stimulusresponse (S-R) associations used in the two tasks may explain the hemispheric discrepancy. In the current motor attention task, we used a rather abstract S-R link requiring a complex process of translation (color to hand of response). The kind of direct $\mathrm{S}-\mathrm{R}$ association used by Siebner et al. (position of hand response) was very spatial in nature and is so automatic that it often interferes with responses when the spatial position of the response is irrelevant to the task that is being performed (see Cho \& Proctor, 2004 for the "Simon effect"). The left and right DLPFC may be differentially involved in the selection of motor responses in tasks with different S-R demands, with the right DLPFC playing a greater role when the movement is specified in a spatially congruent manner, reflecting, perhaps, the role of this area in response inhibition (Aron et al., 2004).

\section{Role of the Posterior Parietal Cortex in Motor and Spatial Attention: A Right Hemispheric Network for Reorienting Attention}

We observed a selective increase in RTs in invalidly cued trials only when the target appeared in the left, as opposed to the right side. Although Rushworth, Krams, et al. (2001) found a similar effect with TMS to the right PPC in a spatial attention task, their effect was present on all invalidly cued trials. This discrepancy might have resulted from differences in the spatial attention tasks used given that our paradigm only had a choice of right versus left locations, positioned more peripherally (i.e., at greater eccentricities).

Another, more likely, explanation for our results relates to evidence of left hemispatial neglect in patients suffering right PPC lesions. Several studies have suggested that the attentional mechanism of the right hemisphere spans both right and left hemispaces, whereas the left hemisphere contributes mainly to contralateral attentional processes (Nobre et al., 1997; Corbetta et al., 1993;
Mesulam, 1981). This would result in left hemispatial neglect in right PPC patients (Heilman \& Van Den Abell, 1980) because the right hemispace might be compensated by the left PPC. Patients may experience contralateral neglect even minutes after a stroke. These few minutes may be crucial for the switch to left-hemisphere control, as revealed in the current results. Thus, "off-line" rTMS may have allowed a rapid reorganization of the brain, such that only a left-side deficit was observed.

\section{Neural Effects of “Off-line" r'TMS Conditioning}

We found a behavioral effect of subthreshold 5 Hz rTMS conditioning that only lasted $10 \mathrm{~min}$ after the end of the stimulation period. This is at odds with electrophysiological studies of the conditioning effects of this rTMS protocol on motor cortical excitability, which have reported effects lasting up to $1 \mathrm{hr}$ (Peinemann et al., 2004; Rizzo et al., 2004). Our results cannot be explained by a generalized TMS effect, as the changes were modulated by task and site of stimulation. In addition, the order of TMS sessions was counterbalanced among subjects who were allocated to each task and site of stimulation randomly. Most of the evidence for the duration of $5 \mathrm{~Hz}$ rTMS is extrapolated from data on motor cortical excitability (Peinemann et al., 2004), so it remains unknown whether the duration of changes induced by rTMS might differ according to the site of stimulation. In the absence of physiological data from the prefrontal or parietal cortices, it is difficult to speculate on the time course of rTMS conditioning effects on the DLPFC or the PPC.

The behavioral effects observed following rTMS conditioning presented in this study point toward a role for the left DLPFC in motor attention and the right PPC in spatial attention. Although these changes suggest a direct effect on the excitability of the stimulated sites, they do not preclude changes in network connectivity that could only be observed with functional neuroimaging techniques. rTMS of $5 \mathrm{~Hz}$ leads to activity changes within entire functional networks, possibly through alterations in effective connectivity of the stimulation site with other regions involved in the task (Pleger et al., 2006; Rounis et al., 2005). A disruption of the DLPFC and the PPC with rTMS might have compromised their outputs to functionally connected areas during reorienting of attention, leading to the behavioral effects observed in this study. Future studies using functional neuroimaging and rTMS, combined with analyses of effective connectivity, might help to better disentangle the roles and interactions of these areas in motor and spatial attentional processes.

\section{Acknowledgments}

This work has been supported by the Brain Research Trust (E. R.), the Medical Research Council (J. C. R.), and the Wellcome Trust (K. Y.). We thank Prof. J. Driver as well as the 
anonymous reviewers and the editor, Prof. R. Ivry, for their helpful suggestions about the design of this study.

Reprint requests should be sent to Elisabeth Rounis, Sobell Department of Motor Neuroscience and Movement Disorders, 8-11 Queen Square, London WC1N 3BG, UK, or via e-mail: e.rounis@ion.ucl.ac.uk.

\section{REFERENCES}

Aron, A. R., Monsell, S., Sahakian, B. J., \& Robbins, T. W. (2004). A componential analysis of task-switching deficits associated with lesions of left and right frontal cortex. Brain, 127, $1561-1573$

Ashbridge, E., Walsh, V., \& Cowey, A. (1997). Temporal aspects of visual search studied by transcranial magnetic stimulation. Neuropsychologia, 35, 1121-1131.

Banich, M. T., Milham, M. P., Atchley, R. A., Cohen, N. J., Webb, A., Wszalek, T., et al. (2000). Prefrontal regions play a predominant role in imposing an attentional "set": Evidence from fMRI. Brain Research, Cognitive Brain Research, 10, 1-9.

Bartolomeo, P., D'Erme, P., Perri, R., \& Gainotti, G. (1998). Perception and action in hemispatial neglect. Neuropsychologia, 36, 227-237.

Bjoertomt, O., Cowey, A., \& Walsh, V. (2002). Spatial neglect in near and far space investigated by repetitive transcranial magnetic stimulation. Brain, 125, 2012-2022.

Brass, M., \& von Cramon, D. Y. (2004). Selection for cognitive control: A functional magnetic resonance imaging study on the selection of task-relevant information. Journal of Neuroscience, 24, 8847-8852.

Cho, Y. S., \& Proctor, R. W. (2004). Influence of multiple spatial stimulus and response codes on orthogonal stimulus-response compatibility. Perceptual Psychophysiology, 66, 1003-1017.

Corbetta, M., Kincade, J. M., Ollinger, J. M., McAvoy, M. P., \& Shulman, G. L. (2000). Voluntary orienting is dissociated from target detection in human posterior parietal cortex. Nature Neuroscience, 3, 292-297.

Corbetta, M., Miezin, F. M., Dobmeyer, S., Shulman, G. L., \& Petersen, S. E. (1991). Selective and divided attention during visual discriminations of shape, color, and speed: Functional anatomy by positron emission tomography. Journal of Neuroscience, 11, 2383-2402.

Corbetta, M., Miezin, F. M., Shulman, G. L., \& Petersen, S. E. (1993). A PET study of visuospatial attention. Journal of Neuroscience, 13, 1202-1226.

Corbetta, M., \& Shulman, G. L. (2002). Control of goal-directed and stimulus-driven attention in the brain. Nature Reviews Neuroscience, 3, 201-215.

Coull, J. T., \& Nobre, A. C. (1998). Where and when to pay attention: The neural systems for directing attention to spatial locations and to time intervals as revealed by both PET and fMRI. Journal of Neuroscience, 18, 7426-7435.

Ellison, A., Schindler, I., Pattison, L. L., \& Milner, D. A. (2004). An exploration of the role of superior temporal gyrus in visual search and spatial perception using TMS. Brain, 127, 2307-2315.

Fierro, B., Brighina, F., Oliveri, M., \& Caltagirone, C. (2000). Contralateral neglect induced by right posterior parietal rTMS in normal subjects. NeuroReport, 11, 1519-1521.

Heilman, K. M., \& Van Den Abell, T. (1980). Right hemisphere dominance for attention: The mechanism underlying hemispheric asymmetries of inattention (neglect). Neurology, 30, 327-330.
Husain, M., Mattingley, J. B., Rorden, C., Kennard, C., \& Driver, J. (2000). Distinguishing sensory and motor biases in parietal and frontal neglect. Brain, 123, 1643-1659.

Jahanshahi, M., \& Rothwell, J. (2000). Transcranial magnetic stimulation studies of cognition: An emerging field. Experimental Brain Resesarch, 131, 1-9.

La Berge, D., LeGrand, D. R., \& Hobbie, R. K. (1969). Functional identification of perceptual and response biases in choice reaction time. Journal of Experimental Psychology, 79, 295-299.

Liu, T., Stolnick, S. D., Serences, J. T., \& Yantis, S. (2003). Cortical mechanisms of feature-based attentional control. Cerebral Cortex, 13, 1334-1343.

MacDonald, P. A., \& Joordens, S. (2000). Investigating a memory-based account of negative priming: Support for selection-feature mismatch. Journal of Experimental Psychology and Human Perceptual Performance, 26, 1478-1496.

Mattingley, J. B., Husain, M., Rorden, C., Kennard, C., \& Driver, J. (1998). Motor role of human inferior parietal lobe revealed in unilateral neglect patients. Nature, 392, 179-182.

Mayr, U., Diedrichsen, J., Ivry, R., \& Keele, S. W. (2006). Dissociating task-set selection from task-set inhibition in the prefrontal cortex. Journal of Cognitive Neuroscience, $18,14-21$.

Mesulam, M. M. (1981). A cortical network for directed attention and unilateral neglect. Annals of Neurology, 10, 309-325.

Mottaghy, F. M., Gangitano, M., Sparing, R., Krause, B. J., \& Pascual-Leone, A. (2002). Segregation of areas related to visual working memory in the prefrontal cortex revealed by rTMS. Cerebral Cortex, 12, 369-375.

Munchau, A., Bloem, B. R., Irlbacher, K., Trimble, M. R., \& Rothwell, J. C. (2002). Functional connectivity of human premotor and motor cortex explored with repetitive transcranial magnetic stimulation. Journal of Neuroscience, 22, 554-561.

Nobre, A. C., Sebestyen, G. N., Gitelman, D. R., Mesulam, M. M., Frackowiak, R. S., \& Frith, C. D. (1997). Functional localization of the system for visuospatial attention using positron emission tomography. Brain, 120, 515-533.

Oliveri, M., Caltagirone, C., Filippi, M. M., Traversa, R., Cicinelli, P., Pasqualetti, P., et al. (2000). Journal of Physiology, 529, 461-468.

Pascual-Leone, A., Valls-Sole, J., Wassermann, E. M., \& Hallett, M. (1994). Responses to rapid-rate transcranial magnetic stimulation of the human motor cortex. Brain, 117, 847-858.

Peinemann, A., Riemer, B., Loeer, C., Quartarone, A., Munchau, A., Conrad, B., et al. (2004). Long-lasting increase in corticospinal excitability after 1800 pulses of subthreshold $5 \mathrm{~Hz}$ repetitive TMS to the primary motor cortex. Clinical Neurophysiology, 115, 1519-1526.

Pleger, B., Blankenburg, F., Bestmann, S., Ruff, C. C., Wiech, K., Stephan, K. E., et al. (2006). Repetitive transcranial magnetic stimulation-induced changes in sensorimotor coupling parallel improvements of somatosensation in humans. Journal of Neuroscience, 26, 1945-1952.

Pollmann, S. (2001). Switching between dimensions, locations, and responses: The role of the left frontopolar cortex. Neuroimage, 14, S118-S124.

Posner, M. I., Walker, J. A., Friedrich, F. J., \& Rafal, R. D. (1984). Effects of parietal injury on covert orienting of attention. Journal of Neuroscience, 4, 1863-1874.

Rizzo, V., Siebner, H. R., Modugno, N., Pesenti, A., Munchau, A., Gerschlager, W., et al. (2004). Shaping the excitability of 
human motor cortex with premotor rTMS. Journal of Physiology, 554, 483-495.

Rounis, E., Lee, L., Siebner, H. R., Rowe, J. B., Friston, K. J., Rothwell, J. C., et al. (2005). Frequency specific changes in regional cerebral blood flow and motor system connectivity following rTMS to the primary motor cortex. Neuroimage, 26, 164-176.

Rounis, E., Stephan, K., Lee, L., Siebner, H., Pesenti, A., Friston, K., et al. (2006). Acute changes in fronto-parietal activity following rTMS over the dorsolateral prefrontal cortex in a cued reaction time task. Journal of Neuroscience, 26, 9629-9638.

Rosenbaum, D. A. (1980). Human movement initiation: Specification of arm, direction, and extent. Journal of Experimental Psychology and Genetics, 109, 444-474.

Rushworth, M. F., Krams, M., \& Passingham, R. E. (2001). The attentional role of the left parietal cortex: The distinct lateralization and localization of motor attention in the human brain. Journal of Cognitive Neuroscience, 13, 698-710.

Rushworth, M. F., Nixon, P. D., Renowden, S., Wade, D. T.,
\& Passingham, R. E. (1997). The left parietal cortex and motor attention. Neuropsychologia, 35, 1261-1273.

Rushworth, M. R., Ellison, A., \& Walsh, V. (2001). Complementary localization and lateralization of orienting and motor attention. Nature Neuroscience, 4, 656-661.

Shallice, T. (1988). From neuropsychology to mental structure. Cambridge, UK: Cambridge University Press.

Siebner, H., Pesenti, A., Rizzo, V., \& Rothwell, J. (2003). Delayed response switching after $5 \mathrm{~Hz}$ repetitive transcranial magnetic stimulation to the right dorsal prefrontal cortex. Neuroimage, 19, 17896. [Available on CD-ROM in issue 2, Suppl. 1].

Snyder, L. H., Batista, A. P., \& Andersen, R. A. (1997). Coding of intention in the posterior parietal cortex. Nature, 386, 167-170.

Steinmetz, M. A., \& Constantinidis, C. (1995). Neurophysiological evidence for a role of posterior parietal cortex in redirecting visual attention. Cerebral Cortex, 5, 448-456.

Stelmach, G. E., \& Teulings, H. L. (1984). Motor programming and temporal patterns in handwriting. Annals of the New York Academy of Sciences, 423, 144-157. 